\title{
Zirconia a Bio-inert Implant Material
}

\author{
S. Kanchana, Dr. Sharmila Hussain \\ Saveetha Dental College \& Hospitals, India
}

\begin{abstract}
Implant ology has become a choice for replacement of missing teeth. Replacements in the anterior zone where aesthetic concern are important were new aesthetic materials are being used. Traditionally Titanium which has been used for past three decades has limitations like hypersensitivity and lack of aesthetics. Zirconia is now being introduced as an aesthetic alternative for implant placement. This paper discusses the properties of zirconia and reviews studies done as an implant material.

Keywords: Zirconia, Mechanical properties, tetragonal-to-monoclinic transformation, Biocompatibility, Animal studies
\end{abstract}

\section{Introduction:}

Implant treatment is currently overriding other prosthetic treatment especially in the case of replacing anterior teeth. Titanium is the material of choice for implants till date. Unfortunately, it is no longer considered as completely bio inert material because of its ability to induce hyper sensitivity reaction. To overcome this limitation zirconia bio ceramics have been recognized as a potential material as an implant because of its biological, mechanical, optical properties and good scientific results in Osseo integration. Due to its transformation toughening capability zirconia holds a unique place in oxides.

\section{Transformation toughening}

Earlier zirconia was applied as a coating material for oral implants in animal investigations. Cranin et al (1) used zirconia flame spray deposition coated vitallium implants in beagle dogs. The results were not satisfactory. So, to improve the integration of zirconia these patients are treated to strengthening and toughening mechanisms.

Zirconia is available in three forms tetragonal, monoclinic and cubic form. When pure zirconium oxide is heated to a temperature of 1470 degree Celsius and 2010 degree Celsius and it is cooled, it transforms from metastable tetragonal form to stable monoclinic from at approximately 1150 degree Celsius. Tetragonal form cannot withstand more stress. When a crack develops tetragonal grains converts immediately to monoclinic from. As the crack propagates sufficient stress develops in the tetragonal structure and the grains around the crack transforms to stable monoclinic form. In this process 3 volume $\%$ of expansion of zirconium dioxide crystals occur, which produces compressive stress around the crack and prevents further propagation of crack (2)(3)(4).

Zirconia ceramics have enhanced toughness because the energy associated with crack propagation is dissipated both in overcoming the compressive stresses and tetragonal to monoclinic transformation. This mechanism is known as Transformation toughening on this basis yttrium-tetragonal zirconia polycrystal is considered to be as high strength Zirconia (5).The transformation of tetragonal to monoclinic form is influenced by temperature, vapor, particle size, micro and macrostructure and concentration of stabilizing oxides (6) .

\section{Martensitic transformation:}

Phase transformation of zirconia is a martensite type. This Phenomenon is named by Osmond and introduced the term "Martensite" (7) .This processes is characterized by a transformation at a certain range of temperature and produce a changes in the shape of the nucleus. The intensity of the martensite transformation is influenced by size, shape, amount of oxide stabilizers, location of particles and difference in co-efficient of thermal expansion. (8)(7).

\section{Zirconia a Biomaterial of choice:}

Introduction: Akagawa et al (9) were the first to report the use of oral implants made of zirconia in dogs. They evaluated loaded and unloaded zirconia dental implants. As a result there was no implant mobility or fracture in both the groups. The bone to implant contact ratio was $81.9 \%$ for the non-loaded implants and $69.8 \%$ for the loaded groups. But, titanium implant group was not included for comparison.

\section{Biocompatibility:}


Biocompatibility of a material refers to the ability of the material to provide derived function without causing any undesirable local or systemic effects in the body i.e., the material should not cause any kind of allergic, toxic (or) inflammatory response.

When it comes to zirconia, release of residues in undetectable and is known to Osseo conductive. Zirconia facilitates bone formation when it comes in contact with it.

\section{Limitations of titanium:}

1. High cost because the amount of processing energy and melting and casting difficulties.

2. Higher elastic modulus compared to bone.

3. Bone attachment is difficult because it do not react with human tissues.

The processing: the molten metal and the hot casting are susceptible to atmospheric contamination. Titanium is very reactive with oxygen and other atmospheric gases. Another problem is the maintenance of good flow over severe changes of dimension or direction with the mold.

The elastic modulus: the elastic moduli and strength of titanium, and its alloys are much higher than those of human bones, which may result in stress shielding and the failure of implants.

The inert behavior: fixation of implants to bone host remains a problem. Titanium has an inert behavior so the body tries to enclose the titanium based implant. However, titanium does not bond directly to bone resulting in micro-movements and leads to loosening of implants (10) (11).

\section{Hypersensitivity reaction}

Evrard et al (12) concluded that inert material like gold and Titanium shows increased rate of allergic reaction in points with history of allergic to metals. To implants may produce toxicity or a type I \& IV hypersensitivity reaction in such points. A titanium hip implant showed a quite strong inflammatory response and was regarded as a contributing factor for failure. Zirconia doesn't produce any hypersensitivity reaction \& is safe to use as an implant material in such patients.

\section{In vitro tests:}

Zirconia, under different physical forms, was tested in vitro onto different cell lines such as fibroblasts, lymphocytes, monocytes, macrophages, and osteoblasts for its toxic potency. Bukat \& coworkers had done (13) early in vitro studies showed higher rate of cytotoxic effects of zirconia due to their wear products than that from Titanium alloys in dose dependent manner. But, authors concluded that zirconia ceramics have low cytotoxicity \&increased cellular growth rate of fibroblasts (14).

In vitro tests on lymphocytes, macro phages and monocyte showed that Titanium\& alumina particles are great inducers of Tumor necrosis factor alpha inflammation marker (15)and showed no effects on human monocytic cell line test.

\section{Osseo integration:}

A process of implant-bone interaction, that finally leads to bone-implant anchorage. Various animal studies have been conducted to demonstrate the Osseo integration and biocompatible nature of zirconia.

In a study conducted by Schultze-Mosgau et al (16) it was found that the interface around zirconia and titanium implants is the same. The biocompatibility test was done by implanting zirconia ceramics in bone and soft tissues. The results show that, only a small inflammatory cell infiltrate has seen, but the implant surface is completely encapsulated by a thin fibrous connective tissue (17).

Antonio scarano et al (18) analyzed the bone response to zirconia implants by implementing them in the rabbit's tibia. A total of twenty implants were retrieved. The percentage of bone contact was calculated using an optical system and from these results authors concluded that all zirconia implants appeared to be

Osseo integrated. Zirconia implants showed presence of newly formed bone around the implant surface along with many osteoblasts in direct contact with the implant. No foreign body reactions inflammation or giant cells are seen.

Hulbert et al (19) investigated the Osseo integration of zirconia by implanting disc and tubes of calcium oxide + zirconium di oxide and calcium oxide + titanium di oxide into rabbit muscles. They concluded that zirconia implant showed no signs of toxic or carcinogenic effects.

Twenty-four screws type zirconia with surface modifications and twenty four titanium implants of same shape and size inserted in the tibia of minipigs a study done by Rita Depprich et al (20). The results showed that animals were normal and showed no signs of infection. On clinical examination they observed new bone formation and circumferential bone tissue formation was detected. Both the titanium and zirconia showed similar Osseo integration and no sign of inflammation. The bone to implant contact increased over period and there were no differences observed between titanium and zirconia. 
B.Stadlinger et al (21) done an animal study to investigate and compare the Osseo integration of zirconia and titanium dental implants. In this study 14 zirconia implants and 7 titanium implants were placed into the mandibles of 7 mini pigs. The zirconia implants were implanted alternatively as submerged and non-submerged. As a result, submerged zirconia implants showed a bone implant contact of 53\% and non - submerged implants showed $48 \%$. Highest bone volume density can be detected in the sub merged zirconia. This study concludes that unloaded zirconia and titanium implants showed comparable amounts of Osseo integration.

A study conducted with sheep pelvis model by J.D. Langhoff et al (22) where six types of dental implants were tested for Osseo integration. One zirconia implant and four titanium implants with surface modifications were implanted and they zirconia implant show high bone implant contact of about $77 \%$ compared to titanium implants. There were no significant differences in Bone- Implant Contact.

Andriotelli et al (23) nine animal studies concluded that zirconia implant have great Osseo integration properties. In conclusion, most of the published data on in vitro studies of zirconia implant reported that zirconia has no cytotoxic effects. In vivo studies show zirconia is considered to be the bio inert material of choice for implant.

\section{In vivo Tests \\ Biocompatibility in soft \&hard tissues:}

Several studies conducted in various animals(24) reported on behavior of zirconia in soft tissues these studies revealed that powders and wear products doesn't produce any local or systemic reaction when injected in mice. So, zirconia in which ever physical form does not induce cytotoxic effects in soft tissues.

Biocompatibility in hard tissue was issued by Helmer \&Driskell (25). This study was conducted by inserting a pellet of stabilized zirconia with $6 \% .1 / 2 \mathrm{O} 3$ into femur of monkeys. They revealed no toxic effects. Bars \& cylinders were also implanted in bones of rats, rabbits \& mice and they induce no systemic or local toxic effects.

Finally, zirconia in its various physical forms induced no toxic effects on hard \& soft tissues from these in vivo tests zirconia is considered to be a biocompatible material of choice.

\section{Perio Integration:}

Zirconia implants creates a low inflammatory response \& presence of angiogenic factors demonstrated by Degidi et al (26) from peri implant soft tissues of zirconia healing cap compared to Titanium.

Bacterial adhesion and plaque accumulation onto zirconia from both in vitro and in vivo studies:

As all natural teeth bacterial growth can also occur in dental implant which may lead to peri implantitis. This is the major factor for failure of dental implant.

Zirconia implant showed low plaque accumulation compared to other materials. During initial stages of plaque formation implant surface properties may play an important role in bacterial adhesion. When it's affected to a greater extend, bacterial adhesion may be due to material's surface roughness (27).

Rimondini et al (28) evaluated the bacterial adhesion on zirconia and titanium through in vivo and in vitro studies. Authors observed that S. Mutants are those showed increased attachment to zirconia compared to titanium in in-vitro studies.

In vivo, however zirconia implants showed minimal bacterial adhesion than titanium .But it revealed no major differences between supra gingival and sub gingival plaque accumulation on zirconia and titanium implant. These finding concludes that zirconia shows reduced early bacterial adhesion compared to titanium implants.

\section{Conclusion:}

Titanium dental implants are still the irreplaceable \& most recommended for patient use. However its adverse reactions are still controversial. Zirconia based on its biocompatible properties \& improved Osseo integration, lesser bacterial adhesion takes importance in new generation of dental implants. From the various in vivo and in vitro studies zirconia seems to be a bio inert material which supports the implementation of this material in dental implant ology.

\section{References:}

[1]. Cranin AN, Schnitman PA, Rabkin SM, Onesto EJ. Alumina and zirconia coated vitallium oral endosteal implants in beagles. J Biomed Mater RES1975: 9: 257-262.

[2]. Morena R, Lockwood PE, Evans AL and Fairhurst CW: toughening of dental porcelain by tetragonal zro2 addition. J Am Ceram soc 69: C75, 1986

[3]. Christel P, Meunier A, Heller M,Torre JP, Peille CN. Mechanical properties and short-term in vivo evaluation of yttrium oxidepartially-stabilized zirconia. J Biomed Mater Res 1989;23:45-61.

[4]. Piconi C, Maccauro G. Zirconia as a ceramic biomaterial. Biomaterials 1999;20:1-25.

[5]. Gupta TK, Lange FF, Bechtold JH. Effect of stress-induced phase transformation on the properties of polycrystalline zirconia containing metastable tetragonal phase. J Mater Sci 1978;13:1464-1470. 
[6]. Kelly, J. R. \& Denry, I. (2008). Stabilized zirconia as a structural ceramic: An overview. Dent Mater, Vol. 24, No. 3, (Mar, 2008) pp. 289-298, ISSN 0109-5641.

[7]. Kelly, P.M.; Francis Rose, L.R. (2002). The martensitic transformation in ceramics-its role in transformation toughening. Prog Mater Sci, Vol. 47, (Mar, 2002) pp. 463-557, ISSN 0079-6425.

[8]. Quinn, J.B.; Sundar, V.; Lloyd, I.K. (2003). Influence of microstructure and chemistry on the fracture toughness of dental ceramics. Dent Mater, Vol. 19, No. 7, (Nov, 2003) pp. 603-611, ISSN 0109-5641.

[9]. Akagawa y, ichikawa Y, Nikai H, Tsuru H. Interface histology of unloaded and early loaded partially stabilized zirconia end osseous implant in initial bone healing. J Prosthet Dent 1993:69:599-604.

[10]. Currey, J. (1998). Mechanical properties of vertebrate hard tissues. Proceedings of the Institution of Mechanical Engineers, Part H: Journal of Engineering in Medicine, Vol.212, No.6, (1998), pp. 399-411, ISSN 09544119.

[11]. Carolos oldani and Alejandro Dominguez. Titanium as a biomaterial for implants.

[12]. Evrard L WD, Parent D. Allergies to dental metals. Titanium: a new allergen. Rev Med Brux. 2010;31(1):44-9

[13]. Bukat A, Fassina P , Greco F et al . GSP made ca-PSZ for biomedical applications. 1990. Pp 355-366

[14]. Raffaelli L, Iommetti PR, Piccioni E, Toesca A, Serini S, Resci F, et al. Growth, viability, adhesion potential, and fibronectin expression in fibroblasts cultured on zirconia or feldspatic ceramics in vitro. Journal of Biomedical Materials Research Part A. 2008;86A(4):959-68

[15]. Sterner T SN, Saxler G, Jakob F, Rader CP. Effects of clinically relevant alumina ceramic, zirconia ceramic and titanium particles of different sizes and concentrations on TNF-alpha release in a human macrophage cell line. Biomed Tech (Berl). 2004;49:340-4.

[16]. Schultze-Mosgau S, Schliephake H, Radespiel-Troger M, Neukam FW. Osseo integration of endodontic end osseous cones: zirconium oxide vs. titanium. Oral Surg Oral Med Oral Pathol Oral Radiol Endod. 2000;89:91-98.

[17]. Ichigawa Y, Akagawa Y, Nikai H, Tsuru H. Tissue compatibility and stability of a new zirconia ceramic in vivo. J Prosthet Dent. 1992;68:322-326

[18]. Antonio Scarano , Fabio Di Carlo , Manlio Quaranta et al . Bone response to zirconia ceramic implant an experimental study in rabbits . J of Oral Implantology feb 2003

[19]. Hulbert SF, Morrison SJ, Klawitter JJ. Tissue reaction to three ceramics of porous and non-porous structures. J Biomed Mater Res. 1972:6:347-374.

[20]. Rita Depprich, Holger Zipprich , Michelle Ommerborn et al . Osseo integration of zirconia implant compared with titanium : an in vivo study. 11 Dec 2008

[21]. B. Stadlinger, M .Hennig, U.Eckelt et al .comparison of zirconia and titanium implant a fetrv a short healing period . A pilot study in mini pigs 2010.

[22]. J.D.Langhoff, K.voelter, D.Scharnweber et al . Comparison of chemically and pharmaceutically modified titanium and zirconium implant surfaces in dentistry: a study in sheep Dec 2008.

[23]. Andreiotelli M, Wenz HJ ,Kohal R.J.Are ceramic implants a viable alternative to titanium implants? A systemic literature review . clinical oral implants research 2009; 20:32-47

[24]. F.P.Koch, D.Weng,S.Kramer et al .Osseo integration of one piece zirconia implants compared with a titanium implant of identical design :a histomorphometric study in the dog . 13 Jan 2010.

[25]. Helmer JC, Driskell TD. Research on Bio ceramics. Symposium on Use of Ceramics as Surgical Implants. South Carolina. Clemson University; 1969.

[26]. Degidi M AL, Scarano A, Perrotti V, Gehrke P, Piattelli A. Inflammatory infiltrate, micro vessel density, nitric oxide synthase expression, vascular endothelial growth factor expression, and proliferative activity in peri-implant soft tissues around titanium and zirconium oxide healing caps. J Periodontology. 2006;77:73-80.

[27]. Nakazato G, Tsuchiya H, Sato M, Yamauchi M. In vivo plaque formation on implant materials. Int J Oral Maxillofac Implants 1989;4:321-26.

[28]. Rimondini L, Cerroni L, Carrassi A, Torricelli P. Bacterial colonization of zirconia ceramic surfaces: An in vitro and in vivo study Int J Oral Maxillofac Implants 2002;17:793-98. 